\title{
Laser Beam Pointing and Stabilization by Fractional-Order PID Control : Tuning Rule and Experiments
}

\author{
Asem Al-Alwan, Xingang Guo, Ibrahima N'Doye and Taous-Meriem Laleg-Kirati \\ \{asem.alwan, xingang.guo,ibrahima.ndoye, taousmeriem.laleg@kaust.edu.sa\}
}

\begin{abstract}
This paper studies the problem of high-precision positioning of laser beams by using a robust Fractional-Order Proportional-Integral-Derivative (FOPID) controller. The control problem addressed in laser beams aims to maintain the position of the laser beam on a Position Sensing Device (PSD) despite the effects of noise and active disturbances. The FOPID controller is well known for its simplicity with better tuning flexibility along with robustness to noise and output disturbance rejections. Thus, a control strategy based on FOPID to achieve the control objectives has been proposed. The FOPID gains and differentiation orders are optimally tuned in order to fulfill the robustness design specifications by solving a nonlinear optimization problem. A comparison to the conventional Proportional-Integral-Derivative (PID) and robust PID is also provided from simulation and experiment set-up. Due to sensor noise, practical PID controllers that filter the position signal before taking the derivative have been also proposed. Experimental results show that the requirements are totally met for the laser beam platform to be stabilized.
\end{abstract}

Index Terms-Laser Beams, PID, Fractional-Order PID (FOPID), Robust PID, process control, iterative optimization, robust control.

\section{INTRODUCTION}

Laser is a device that emits a beam of light by stimulated emission of photons from excited atoms or molecules. It has been used in different applications such as communication, surgical use, printing, bar code scanning, laser cutting and many other industrial applications. The need for high accuracy pointing is considered as a main challenge for these systems. This is due to the difficulty of reaching the target also to the point and the vibration of the pointing system in presence of external disturbances [1]. The work presented in this paper is to design a robust controller that reduces the active disturbance and minimizes the variations, which cause the laser beam pointing errors.

Several control techniques have been combined with a feedback and/or feedforward control techniques, estimation and/or identification methods such as PID and robust PID controllers [1], [2], passive control [3]-[5], adaptive control techniques or more advanced adaptive techniques such as [6]-[11]. PID controllers with a beam-stabilized optical switch have been proposed in [2], [12] to stabilize a beam at

Asem Al-Alwan, Ibrahima N'Doye and Taous-Meriem Laleg-Kirati are with Computer, Electrical and Mathematical Sciences and Engineering Division (CEMSE), King Abdullah University of Science and Technology (KAUST), Thuwal 23955-6900, Saudi Arabia.

Xingang Guo is with Northeastern University at Qinhuangdao and Computer, Electrical and Mathematical Sciences and Engineering Division (CEMSE), King Abdullah University of Science and Technology (KAUST). a desired angle and maximize the optical power detected by a photodiode using a voice-coil motor actuator. Such studies have showed that the PID controllers can be a good method of stabilizing the laser beam with minimal switching time. Nevertheless such standard control strategies fail to provide good performance particularly under vibration conditions of the pointing systems due to active disturbances.

In this study, the FOPID controller is introduced as an alternative robust control strategy to the PID control [13], [14], [15]. The conventional PID controller is one of the most used controller in industry for closed control-loops thanks to its simplicity in real time implementation. The design and tuning of such a controller has been widely covered and still represents an active field of research especially for industrial plants subject to external disturbances (see [13], [15]). Indeed, due to the significant variations in the amplitude vibration affected by the external disturbances, satisfactory performance covering the total range of disturbances is difficult to reach with a conventional PID without an external compensation. Thereafter, it is desirable to design robust control strategies without additional computational effort.

The FOPID controller is a generalization of the PID controller by considering fractional derivatives and integral actions [16]. In recent years, fractional calculus has gained significant attention in various applications in engineering and science. This interest is driven by the possibility to obtain accurate mathematical models of real objects, especially for those presenting memory, hereditary, or self-similarity properties [16]. Besides of modeling, the fractional calculus has been applied for control design. The FOPID offers better tuning flexibility thanks to the additional differentiation orders allowing for better control performance and robustness. Several studies have addressed the properties of the FOPID and compared its performance to the conventional PID under different contexts (see, e.g., [17]-[23]). Different tuning procedures for the FOPID have been proposed in the literature where the main objective is to optimally tune the gains and the differentiation orders. Examples of these tuning procedures include: the procedure presented in [17], which is based on solving a set of nonlinear equations. In [18] and [19] a tuning rule for a fractional-order controller with application to motion systems is given. Genetic algorithms are used in [20], [24] to design a FOPID controller. Another class of fractional controllers is proposed by [21]. This class of controllers is more appropriate to ensure robust- 
ness of the closed-loop to static gain variations with a conventional CRONE template [22]. Some efficient design techniques have also been proposed and are summarized in [17], [23]. In this paper, we design a FOPID control to reduce the active disturbance and minimize dynamic laser beam pointing errors. The gains and differentiation orders are tuned by solving a nonlinear optimization problem. The requirements to be satisfied by the controller are expressed in order to fulfill some frequency-domain design specifications. The performance of the FOPID is evaluated by comparison to the robust and conventional PID controllers through simulations and experiments. Moreover, robustness analysis is performed with respect to high frequency noise rejection and output disturbance rejection through simulations. Finally, the controllers are validated through experiments.

This paper is organized as follows. In section II, some preliminary results about fractional control are presented. In section III, the laser beam system is described and the control problem is introduced. In section IV, the design and the tuning procedure of the FOPID controller are given. Numerical simulation and experimental results are illustrated and discussed in section $\mathrm{V}$ followed by concluding remarks in section VI.

\section{PREliminaries}

Fractional calculus is a generalization of differential and integral operators to a non-integer order fundamental operator ${ }_{0} D_{t}^{\alpha}$ where $\alpha$ is the operator order and $t$ denotes the limit of the operation. Several definitions of this fractional integro-differential operator exist. However, we restrict our attention to one particular definition, which is used throughout this work for evaluating fractional-order systems. It is the Grünwald-Letnikov definition ([16], [23]).

Definition 1: The Grünwald-Letnikov definition is given as follows:

${ }_{0} D_{t}^{\alpha} f(t)=\frac{d^{\alpha} f(t)}{d t^{\alpha}}=\lim _{t \rightarrow 0} h^{-\alpha} \sum_{j=0}^{\left[\frac{t}{n}\right]}(-1)^{j}\left(\begin{array}{c}\alpha \\ j\end{array}\right) f(t-j h)$,

with $n \in \mathbb{N}, \alpha \in \mathbb{R}^{+}$, [.] denotes the integer part, $t=k h$ is the number of steps, and $h$ the step size.

Assuming zero initial conditions, the Laplace transform of the fractional derivative with $\alpha \in \mathbb{R}^{+}$, is given by:

$$
\int_{0}^{\infty} e^{-s t}{ }_{0} D_{t}^{\alpha} f(t)=s^{\alpha} F(s)
$$

where $s=j \omega$ is the Laplace transform variable. Thus a fractional-order differential equation can be expressed in transfer function form in the frequency domain as follows:

$$
P(s)=\frac{b_{m} s^{\beta_{m}}+b_{m-1} s^{\beta_{m-1}}+\cdots+b_{0} s^{\beta_{0}}}{a_{n} s^{\alpha_{n}}+a_{n-1} s^{\alpha_{n-1}}+\cdots+a_{0} s^{\alpha_{0}}} .
$$

\section{CONTROL PROBLEM FORMULATION}

The principle of laser beam stabilization is demonstrated in Fig. 1. The laser beam source is subject to various disturbances. The beam is first directed to a fast-steering mirror (FSM), that rotates about a pivot. And then the laser beam is reflected from the FSM to a beam splitter. The majority of the beam is directed to the target and a fraction of the beam is directed to a position sensing detector (PSD). PSD measures how far the beam has displaced from the target and sends feedback signal to the control system. Then the control system sends signal to the FSM such that the beam remains stable on the target.

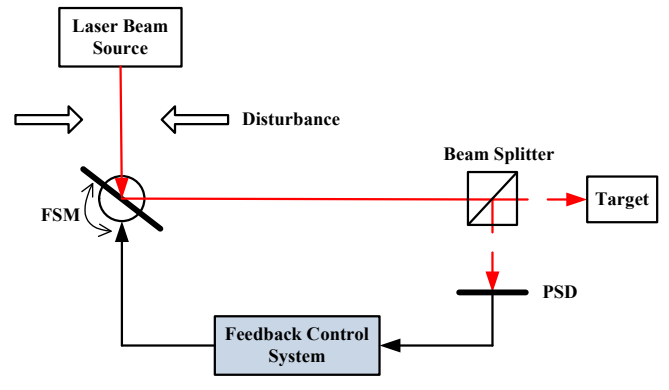

Fig. 1. Laser beam stabilization principle

The transfer function of the open-loop voltage-to-position of the system is given as follows [1]:

$$
P(s)=\frac{K}{s(T s+1)}
$$

where $K=2200 \mathrm{~mm} /(\mathrm{V} . \mathrm{s})$ is the open-loop steady-state gain and $T=0.005 \mathrm{~s}$ is the open-loop time constant.

\section{FOPID CONTROL DESIGN}

The transfer function corresponding to the parallel form of the FOPID where the integral component is of order $\lambda$ and the derivative component is of order $\mu$ has the following form in the Laplace domain:

$$
C(s)=K_{p}+\frac{K_{i}}{s^{\lambda}}+K_{d} s^{\mu}, \quad 0<(\lambda, \mu)<1,
$$

where $K_{p}$ is the proportional gain, $K_{i}$ and $K_{d}$ are the integration and the differentiation gains consecutively.

Several methods for the FOPID controller tuning have been proposed (see [17], [23], [25], [26], [27] and references therein). Numerical optimization based methods are regularly used to solve this problem [27].

In this study, our objective is to design a FOPID control based on numerical optimization in order to study its performance in the control of laser beams under active disturbance. The tuning procedure is based on satisfying a set of equations derived to achieve the control objectives under the frequencydomain robustness constraints. Frequency domain based design of the FOPID controller was first proposed by Monje et al. [17] resorting to a constrained optimization problem.

We consider the fractional orders for the integral and the derivative actions $\lambda$ and $\mu$ of the FOPID controller to be such that $0<(\lambda, \mu)<1$. If $P(s)$ is the model of the process plant, then the objective is to find a controller $C(s)$, such that the open loop system $G(s)=C(s) P(s)$ would meet the following design specifications:

- Gain cross-over frequency specification

$$
\left|G\left(j \omega_{g c}\right)\right|_{d B}=\left|C\left(j \omega_{g c}\right) P\left(j \omega_{g c}\right)\right|_{d B}=0 \mathrm{~d} B .
$$

- Phase margin specification

$$
\arg \left[G\left(j \omega_{g c}\right)\right]=\arg \left[C\left(j \omega_{g c}\right) P\left(j \omega_{g c}\right)\right]=-\pi+\phi_{m},
$$

where $\phi_{m}$ is related to the damping of the system. 
- Robustness to gain variation

$$
\left(\frac{\mathrm{d}}{\mathrm{d} \omega}\left(\arg \left[G\left(j \omega_{g c}\right)\right]\right)\right)_{\omega=\omega_{g c}}=0 .
$$

This condition forces the phase to be flat at $\omega_{g c}$ or almost constant within an interval around $\omega_{g c}$.

- Complementary sensitivity specification

$$
\begin{gathered}
|T(j \omega)|=\left|\frac{C(j \omega) P(j \omega)}{1+C(j \omega) P(j \omega)}\right|_{\mathrm{d} B} \leqslant A \mathrm{~d} B \quad \forall \omega \geqslant \omega_{t} \\
\Longrightarrow\left|T\left(j \omega_{t}\right)\right|=A \mathrm{~d} B
\end{gathered}
$$

where $A$ is the magnitude or noise attenuation for frequencies $\omega \geqslant \omega_{t} \mathrm{rad} / \mathrm{s}$.

- Sensitivity specification

$$
\begin{gathered}
|S(j \omega)|=\left|\frac{1}{1+C(j \omega) P(j \omega)}\right|_{\mathrm{d} B} \leqslant B \mathrm{~d} B \quad \forall \omega \leqslant \omega_{s} \\
\Longrightarrow\left|S\left(j \omega_{s}\right)\right|=B \mathrm{~d} B
\end{gathered}
$$

where $B$ is the magnitude or load disturbance suppression for frequencies $\omega \leqslant \omega_{s} \mathrm{rad} / \mathrm{s}$.

- Elimination of steady-state error: the steady-state error of the closed loop system automatically gets canceled with the introduction of the fractional integrator.

The five parameters $\left(K_{p}, K_{i}, K_{d}, \lambda, \mu\right)$ of the FOPID controller (5) can now be tuned using the five specifications (6)-(10). Here, the numerical optimization algorithm, namely of Nelder-Mead's simplex method, has been implemented using the Matlab function fmincon. This is a local optimization algorithm which depends on initial estimates of the provided parameters. To ensure that the local minimum is reached by the optimization process, we combine two optimization algorithms. We first use the genetic algorithms as an initial search method which provides the initial guess to the fmincon algorithm.

\section{Simulation AND EXPERIMENTAL SET-UP}

This section evaluates the designed FOPID performance for the closed-loop disturbance-to-position of the laser beam stabilizer. Then, the FOPID is implemented for the laser beam stabilizer platform in closed-loop under active disturbance voltage.

The design specifications required for the laser beam system are:

- gain crossover-frequency, $\omega_{g c}=1000 \mathrm{rad} / \mathrm{s}$;

- phase margin, $\phi_{m}=60^{\circ}$;

- robustness to variations in the gain of the plant must be fulfilled;

- noise rejection: $|T(j \omega)| \leqslant-30 \mathrm{~d} B, \forall \omega \geqslant \omega_{t}=2 \times$ $10^{4} \mathrm{rad} / \mathrm{s}$;

- sensitivity function: $|S(j \omega)| \leqslant-100 \mathrm{~d} B, \forall \omega \leqslant \omega_{s}=$ $0.1 \mathrm{rad} / \mathrm{s}$

\section{A. Simulation set-up}

The conventional PID controller gains are calculated based on disturbance rejection PID method [1] :

$$
K_{p}=0.590, K_{i}=0.295, K_{d}=0.00186 .
$$

The next step is to use the FOPID controller optimization algorithm that minimizes the disturbance-to-position magnitude response. We propose two different sets of parameters depending on the tuning rules to test the closedloop performance. Two parameters sets have been generated using the constrained optimization algorithm. The first set is the result of the optimization of the five parameters (gains and fractional orders) leading to an FOPID controller. The second set has been derived by optimizing the gains only while considering integer-order for the integration and the derivation leading to an robust PID controller.

The optimization procedure for both sets has been run by setting the following ranges for the orders:

$$
\lambda=[0.5 ; 1] \text { and } \mu=[0.78 ; 1] \text {. }
$$

The selection of ranges can be easily derived from considering the behavior of the fractional-order control actions in time domain.

The genetic algorithm is first performed and the obtained results are used as the initial search vector for fmincon algorithm. Then, the following FOPID controller parameters are obtained from the two cases:

- Optimization of all parameters (FOPID):

$$
\begin{aligned}
& K_{p}=0.85006, \quad K_{i}=1.99247, \quad K_{d}=0.01002, \\
& \lambda=0.88395, \quad \mu=0.7806 .
\end{aligned}
$$

- Optimization of the gains only (Robust PID):

$$
K_{p}=0.7854, K_{i}=1.8456, K_{d}=0.001, \lambda=\mu=1 .
$$

The resulting three controllers are tested in closed-loop disturbance-to-position for laser beam system (4). The closed-loop simulation responses of the laser beam system using PID, robust PID and Fractional-Order PID controllers for an input disturbance sine signal of $10 \pi \mathrm{Hz}$ frequency and $0.8 \mathrm{~mm}$ amplitude is shown in Fig. 2. The response of the robust and conventional PID controllers shows that the high amplitude vibration of the laser beam reduced to an amplitude of $15 \times 10^{-2} \mathrm{~mm}$ and $20 \times 10^{-2} \mathrm{~mm}$ while the amplitude of the fractional-order PID controller decreased to about $12 \times 10^{-2} \mathrm{~mm}$. From these results, we can conclude that the FOPID controller presents better performance compared to the robust PID and PID controllers in terms of output position errors. The comparative performance evaluation has been also studied in the frequency domain. The Bode diagrams of the open-loop system $G(s)=C(s) P(s)$ are shown in Fig. 3. As it can be seen, the gain crossover frequency specification $\omega_{g c}=1000 \mathrm{rad} / \mathrm{s}$ and the phase margin specification $\phi_{m}=61^{\circ}$ are fulfilled for the FOPID controller. Besides, the phase of the system is forced to be flat at $\omega_{g c}$ and hence to be almost constant within an interval around $\omega_{g c}$.

To assess the robustness of the system response with respect to high frequency noise rejection and output disturbance, we have explored the magnitudes of the functions $T(j \omega)$ and $S(j \omega)$ by analyzing their Bode diagrams as shown in Figs. 4 and 5 respectively. As it can be observed, $|T(j \omega)|_{d B} \leqslant$ $-30 \mathrm{~d} B, \forall \omega \geqslant \omega_{t}=2 \times 10^{4} \mathrm{rad} / \mathrm{s}$, and $|S(j \omega)|_{d B} \leqslant$ $-100 \mathrm{~d} B \forall \omega \leqslant \omega_{s}=0.1 \mathrm{rad} / \mathrm{s}$. We can conclude that both controllers fulfill the specifications but the FOPID controller tends to have more robustness than the PID controller as we 


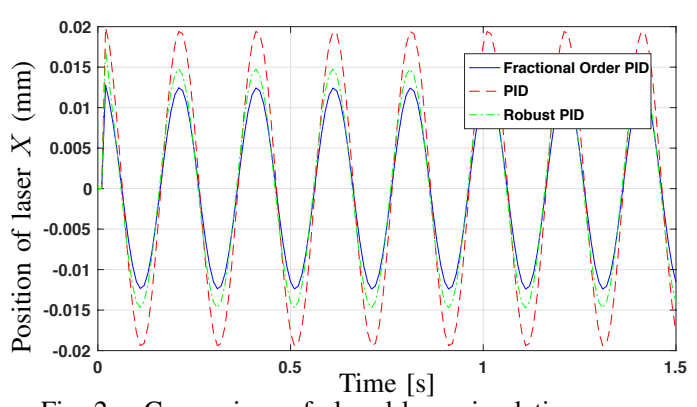

Fig. 2. Comparison of closed-loop simulation responses.

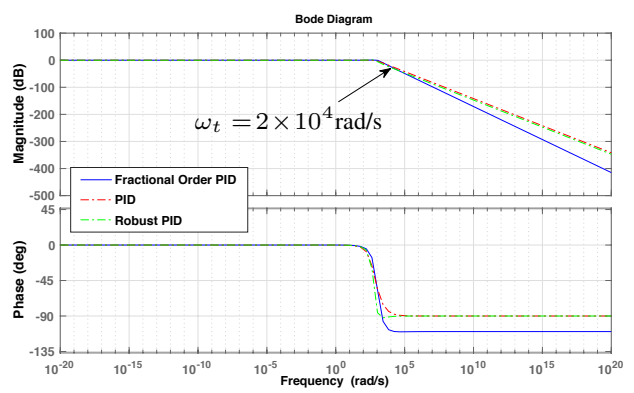

Fig. 4. Bode diagrams of $T(j \omega)$ (noise rejection).

go far from $\omega_{t}$. It means that the FOPID controller has a slighty better robustness than the PID controllers presenting a very satisfactory high frequency noise rejection and output disturbance rejection.

From the overall results presented above, we observe clearly that FOPID controller is more effective and robust than the conventional and robust PID controllers for stabilizing the vibration of the laser beam with relatively smaller amplitude in spite of varying disturbances imposing hard constraints on the closed-loop performance.

\section{B. Ideal and practical experimental set-up}

The laboratory plant used in this work is a laser beam stabilizer system shown in Fig. 6. The plant is conveniently connected to the PC via Quanser Personality Intelligent Data (QPID) acquisition board and Peripheral Component Interconnect (PCI) board for data transfer. MATLAB/Simulink library for real-time control of the plant which uses the WinCon application from Quanser, to communicate with the data acquisition card is available [1].

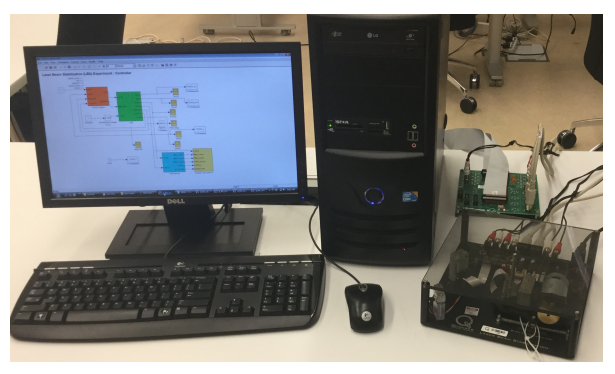

Fig. 6. Laboratory plant

The laser beam stabilization consists of a stationary laser source for providing the laser beam, a FSM which acts as a voice coil mounted on a vibrating platform, a DC motor for subjecting the platform to active disturbance and an amplifier [1]. The amplifier provides the voltage or maximum power

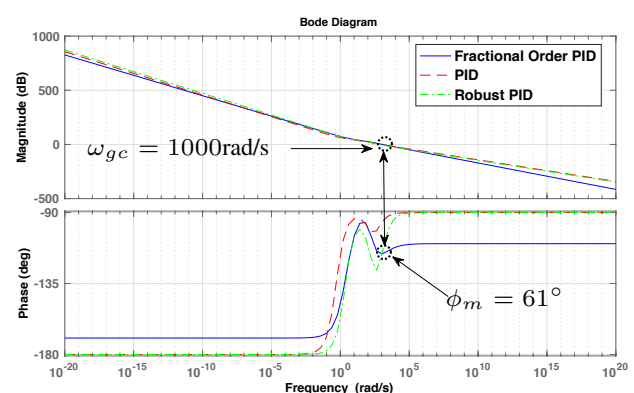

Fig. 3. Bode diagrams of the open-loop system $G(s)=C(s) P(s)$.

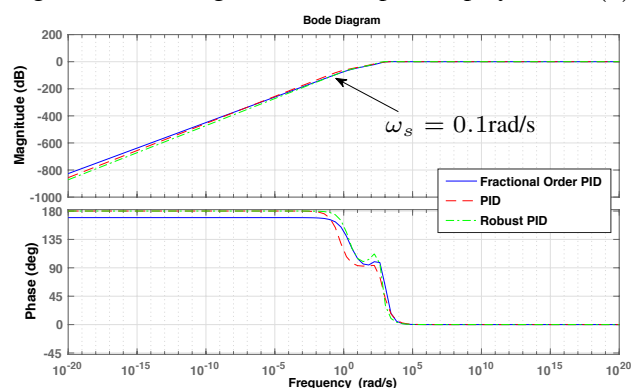

Fig. 5. Bode diagrams of $S(j \omega)$ (output disturbance rejection).

that is used to the actuator by the Digital-to-Analog convertor (D/A) and also magnifies the signal from the position sensing device to the Analog-to-Digital converter. The QPID acts as a data acquisition board and also acts as a Digital-to-Analogto-Digital convertor (D-A-D), thus it digitizes the analog position signal of the laser beam measured by the position sensor for the computer and also converts digitized control signal from the computer to analog form for the actuator [1]. The proposed PID, robust and fractional-order PID controllers aimed to stabilize the vibrations induced into the beam using the FSM and feedback from the position sensor. The experimental platform has been used to test the fractional-order controllers designed by the optimization tuning method proposed previously. An ideal PID controller with no filtering is first derived. Next, a practical PID controller that uses filtering in its control is built.

1) Ideal experimental set-up: In practice fractional-order operator approximations are often used to overcome the problem of infinite memory requirement that the fractionalorder systems possess [28]. Oustaloup filter method and its modification [29], [30] provide a good approximation technique where the fractional derivatives are approximated by integer-oder ones. Thus, the fractional integral and derivative parts have been implemented using the Oustaloup continuous approximation of the fractional integrator [29], [30] choosing a frequency band from 0.001 to $10^{5} \mathrm{rad} / \mathrm{s}$ and an order of the approximation equal to 10 (number of poles and zeros). Once the continuous fractional controller is obtained, it is discretized by using the Tustin rule discretization technique. Figs. 7 - 9 illustrate important characteristics of the experimental performance of the proposed controllers. Fig. 7 shows the plot of experimental response of the PID controller that is switched from an automated Seeker Mode (SM) to closedloop after $t=2 \mathrm{sec}$. The automated seeker system swivels the voice-coil until the PSD receives a signal, temporarily 
stabilizes the beam in the center and then locks the voicecoil into that position. The PID controller sustains a relatively amplitude of $0.1 \mathrm{~mm}$ (see Fig. 7). However this vibration is minimized in Fig. 8 to an amplitude of $0.06 \mathrm{~mm}$ for the robust PID controller. The response of the fractionalorder PID controller in Fig. 9 presents a small amplitude of approximately less than $0.04 \mathrm{~mm}$. Fig. 10 shows the experimental responses when switching from PID to robust PID and fractional-order PID controllers. From the obtained experimental results, it is clear that the designed FOPID outperforms the conventional and robust PID controllers.

Further comparative details with the Root Mean Square (RMS) values in the PID, robust PID and Fractional-Order PID of the beam displacement are given in Table I. These RMS values quantify the effect of the closed-loop control with respect to the proposed controllers and it permits us to conclude on the performance of the control strategy. Table I shows that the Fractional-Order PID controller reduces the axis $X$ output error by more than $8 \%$ from the output error to the robust PID controller and $45 \%$ from the ideal PID controller. The plots in Fig. 11 show zoomed view of the practical controllers over the time interval $[19.5-20.5]$ seconds. Fig. 12 shows representative sample trajectories of the laser image on the PSD in the experiment over two different time intervals. From the obtained experimental results, it is clear that the designed FOPID controller outperforms the conventional and robust PID controllers.

TABLE I

RMS OUTPUT POSITION ERRORS

\begin{tabular}{|c||cc|}
\hline Controller & RMS & Interval \\
\hline PID & 0.0295 & $2-25 \mathrm{sec}$. \\
\hline Robust PID & 0.0176 & $2-25 \mathrm{sec}$. \\
\hline Fractional Order PID & 0.0160 & $2-25 \mathrm{sec}$. \\
\hline
\end{tabular}

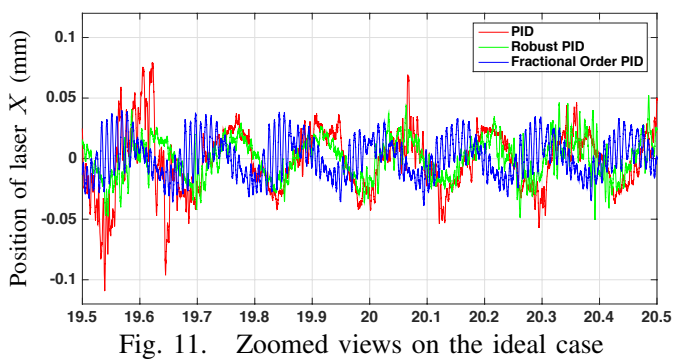

2) Practical experimental set-up: Due the sensor noise, we propose to design practical PID controllers that filter the position signal before taking the derivative. Figs. 13 - 15 show the experimental performance of the proposed controllers. Fig. 13 shows the plot of experimental response of the PID controller that is switched from an automated Seeker Mode (SM) to closed-loop after $t=2$ sec. In Fig. 13, the practical PID controller maintains a relatively amplitude of $0.04 \mathrm{~mm}$. However this vibration is reduced in Fig. 14 to an amplitude of $0.03 \mathrm{~mm}$ for the robust PID controller. The response of the fractional-order PID controller in Fig. 15 presents a small amplitude of approximately less than
$0.02 \mathrm{~mm}$. Fig. 16 shows the experimental responses when switching from practical PID to robust PID and fractionalorder PID controllers. Table II shows that the practical Fractional-Order PID controller reduces the axis $X$ output error by more than $34 \%$ from the output errors to the robust PID controller and $60 \%$ from the output errors of the PID controller. The plots in Fig. 17 show zoomed view of the practical controllers over the time interval [22-23] seconds. Fig. 18 shows representative sample trajectories of the laser image on the PSD in the experiment over two different time intervals. From the obtained practical experimental results, it is clear that the designed FOPID outperforms the practical conventional and robust PID controllers.

TABLE II

RMS OUTPUT POSITION ERRORS

\begin{tabular}{|c||cc|}
\hline Controller & RMS & Interval \\
\hline PID & 0.0254 & $2-25 \mathrm{sec}$. \\
\hline Robust PID & 0.0141 & $2-25 \mathrm{sec}$. \\
\hline Fractional Order PID & 0.0092 & $2-25 \mathrm{sec}$. \\
\hline
\end{tabular}

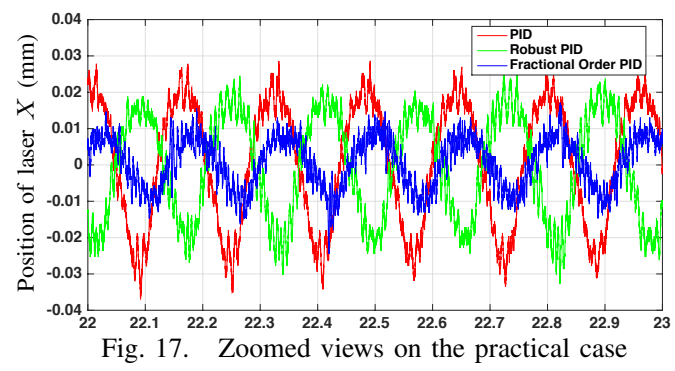

\section{CONCLUSION}

In this paper, a fractional-order PID has been proposed to stabilize a laser beam such that the incident laser beam of the FSM is reflected to the center of the position sensor even in the presence of active disturbance and noise. The performance of the presented FOPID controller has been evaluated in closed loop and it has been compared to the conventional PID and robust PID controllers. The simulation results have shown that the FOPID controller is more effective and robust than the PID and robust PID controllers, while it preserves its simplicity of implementation. Moreover, experimental results show that the FOPID controller can achieve better performance results highlighting the effectiveness of the proposed controllers in terms of robustness.

\section{ACKNOWLEDGMENT}

Research reported in this publication has been supported by the King Abdullah University of Science and Technology (KAUST). 


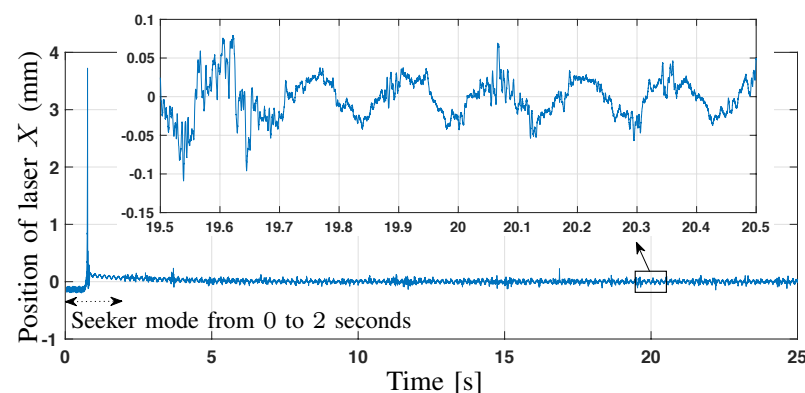

Fig. 7. Response of real laser beam system with PID controller.

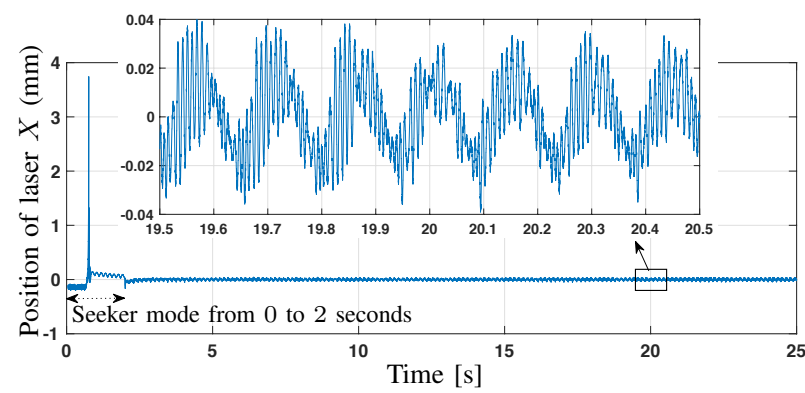

Fig. 9. Response of real laser beam system with Fractional-Order PID (FOPID) controller.

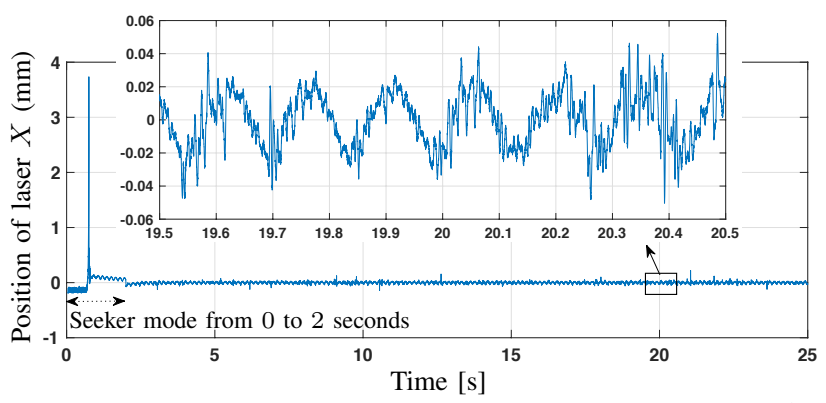

Fig. 8. Response of real laser beam system with Robust PID (RPID) controller.

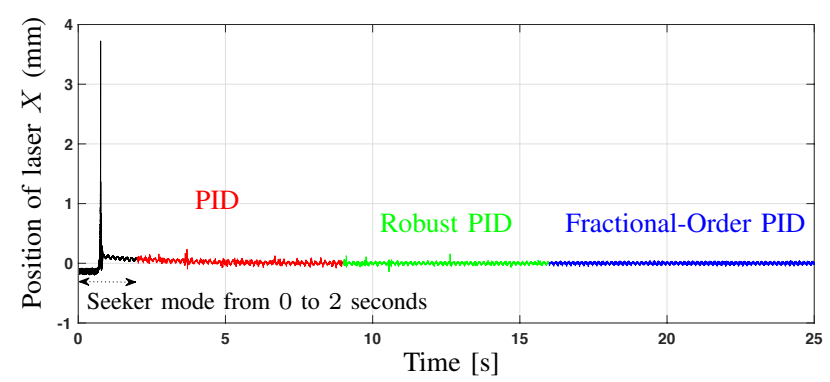

Fig. 10. Experimental responses when switching from PID to robust PID and Fractional Order PID controllers.
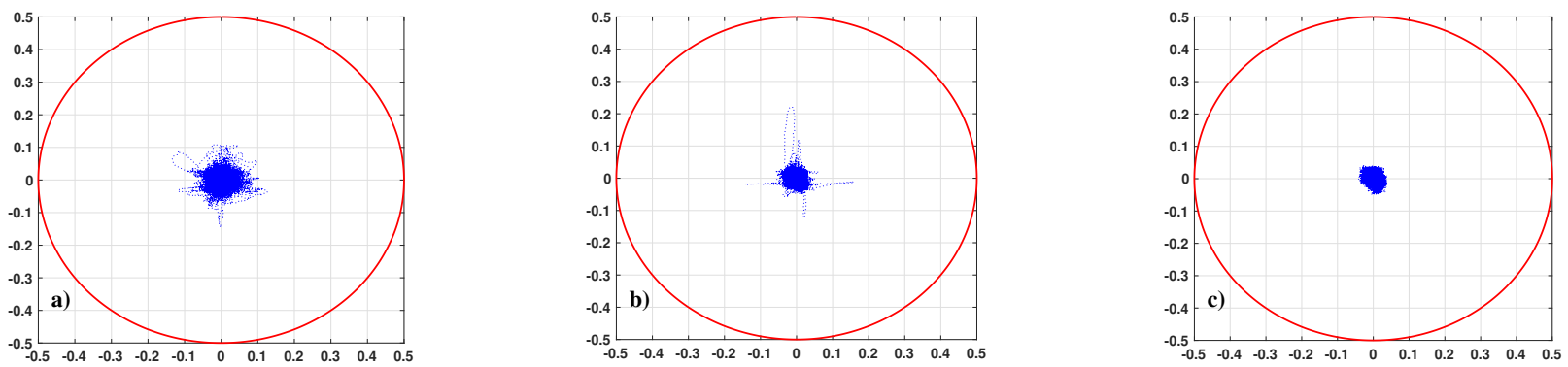

Fig. 12. Screen shots of the laser-beam trajectories on the PSD over two different time intervals : a) PID controller vs PID controller; b) Robust PID controller vs Robust PID controller; c) Fractional-Order PID controller vs Fractional-Order PID controller.

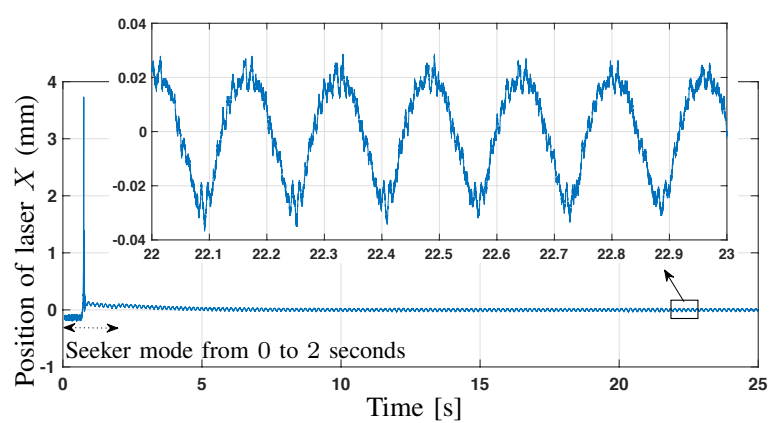

Fig. 13. Response of real laser beam system with PID controller.

\section{REFERENCES}

[1] Quanser, Laser Beam Stabilization Instructor Manual. Quanser Speciality Experiment Series: LBS Laboratory Workbook, 2010.

[2] K. A. Konadu, S. Yi, W. Choi, and T. Abu-Lebdeh, "Robust positioning of laser beams using proportional integral derivative and based observer-feedback control," American Journal of Applied Sciences, vol. 10, no. 4, pp. 374-387, 2013.

[3] O. Zai, "On the control aspect of laser frequency stabilization," in Proc. IEEE American Contr. Conf., pp. 1628-1629, 1992.

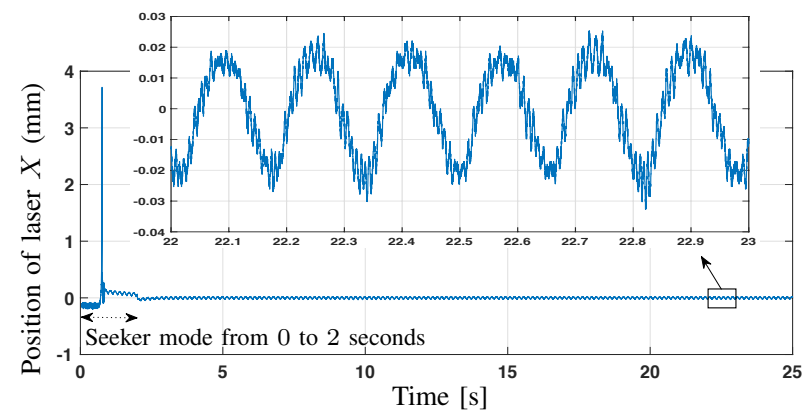

Fig. 14. Response of real laser beam system with Robust PID (RPID) controller.

[4] M. Bodson, A. Sacks, and P. Khosla, "Harmonic generation in adaptive feedforward cancellation schemes," IEEE Trans. Aut. Contr., vol. 39, pp. 1939-1944, 1994.

[5] J. Anderson and T. Sarkodie-Gyan, "Mechanical design and modeling of an inertial reference unit for laser beam stabilization," in World Automation Congress, (Seville, Spain), pp. 389-396, 2004.

[6] N. O. P. Arancibia, S. Gibson, and T. C. Tsao, "Adaptive control of mems mirrors for beam steering," in ASME International Mechanical Engineering Congress and Exposition, (American Society of Mechanical Engineers), pp. 71-80, 2004. 


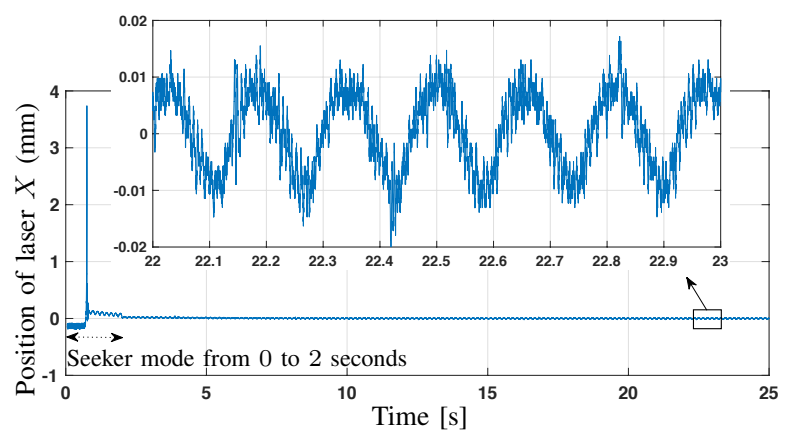

Fig. 15. Response of real laser beam system with Fractional-Order PID (FOPID) controller.

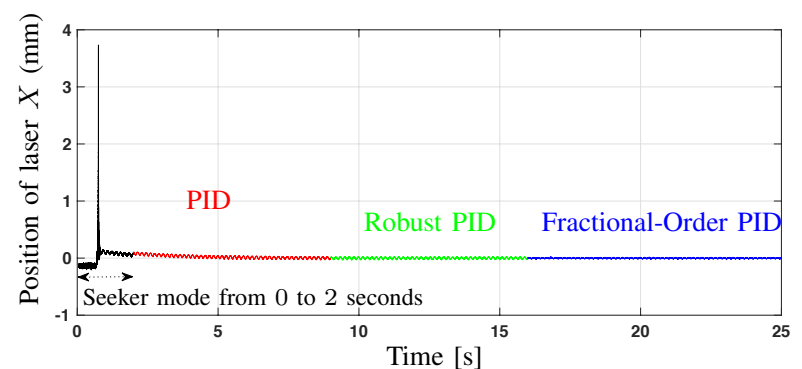

Fig. 16. Experimental responses when switching from PID to robust PID and Fractional Order PID controllers.
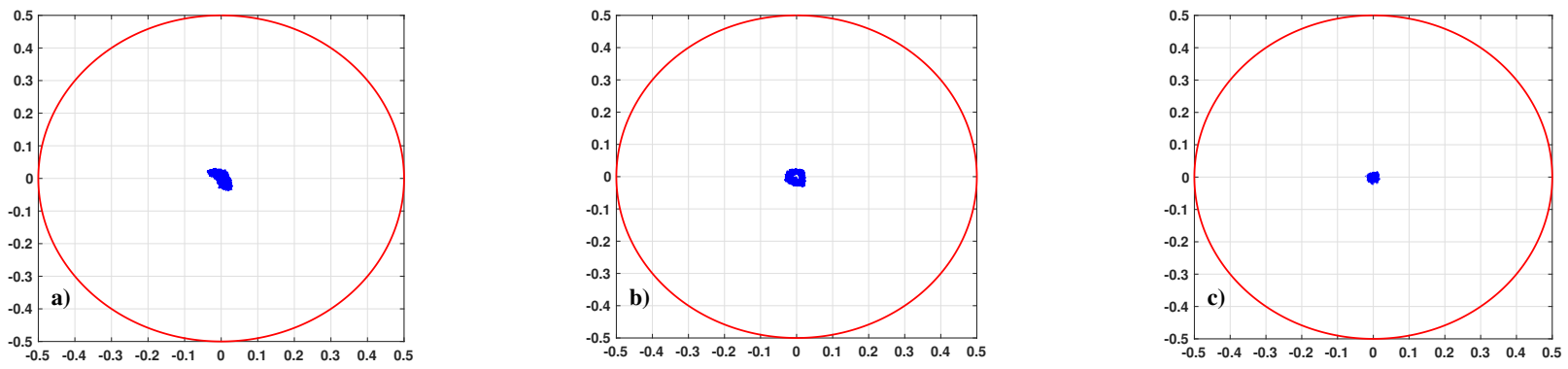

Fig. 18. Screen shots of the laser-beam trajectories on the PSD over two different time intervals : a) PID controller vs PID controller; b) Robust PID controller vs Robust PID controller; c) Fractional-Order PID controller vs Fractional-Order PID controller.

[7] L. Chi-Ying, C. Yen-Cheng, T. Tsu-Chin, and S. Gibson, "Laser beam tracking by repetitive and variable-order adaptive control," in Proc. IEEE American Contr. Conf., (Seattle, WA, USA), pp. 2371-2376, 2008.

[8] T. Richard, J. O'Brien, and R. J. Watkins, "Adaptive $\mathcal{H}_{\infty}$ vibration control," in Dynamic Systems and Control Conference and Bath/ASME Symposium on Fluid Power and Motion Control, (Arlington, Virginia, USA), pp. 181-188, 2011.

[9] T. Tsu-Chin, J. S. Gibson, K. C. Chiu, and C. Shean-Jen, "Adaptive control for focusing of optical drive read/write heads," in Proc. IEEE American Contr. Conf., (San Francisco, CA, USA), pp. 588-593, 2011.

[10] N. O. Perez-Arancibia, J. S. Gibson, and T. Tsu-Chin, "Frequencyweighted minimum-variance adaptive control of laser beam jitter," in IEEE/ASME Trans. Mechatron., vol. 14, pp. 337-348, 2009.

[11] N. O. Perez-Arancibia, J. S. Gibson, and T. Tsu-Chin, "Laser beam pointing and stabilization by intensity feedback control," in Proc. IEEE American Contr. Conf., (St. Louis, MO, USA), pp. 2837-2842, 2009.

[12] T. Landolsi, R. Dhaouadi, and O. Aldabbas, "Beam stabilized optical switch using a voice-coil motor actuator," Journal of the Franklin Institute, vol. 348, no. 1, pp. 1-11, 2011.

[13] K. Astrom and T. Hagglund, "The future of PID control," Control Engineering Practice, vol. 9, no. 11, pp. 1163-1175, 2001.

[14] W. Ho, K. Lim, C. Hang, and L. Ni, "Getting more phase margin and performance out of PID controllers," Automatica, vol. 35, pp. 1579$1585,1999$.

[15] K. Astrom, T. Hagglund, C. C. Hang, and W. K. Ho, "Automatic tuning and adaptation for PID controllers-a survey," Control Engineering Practice, vol. 9, no. 4, pp. 699-714, 1993.

[16] I. Podlubny, Fractional Differential Equations. New York: Academic Press, 1999.

[17] C. Monje, B. Vinagre, V. Feliu, and Y. Chen, "Tuning and autotuning of fractional order controllers for industry applications," Control Engineering Practice, vol. 16, no. 7, pp. 798-812, 2008.

[18] H. S. Li, Y. Luo, and Y. Q. Chen, "A fractional order proportional and derivative (FOPD) motion controller: Tuning rule and experiments," IEEE Trans. Contr. Syst. Techn., vol. 18, pp. 516-520, 2010.

[19] J. Lu and G. Chen, "Robust stability and stabilization of fractionalorder interval systems: an LMI approach," IEEE Trans. Aut. Contr., vol. 54, pp. 1294-1299, 2009.
[20] J. Y. Cao, J. Liang, and B. G. Cao, "Optimization of fractional order PID controllers based on genetic algorithms," in International Conference on Machine Learning and Cybernetics, (Guanzhou, China), pp. 5686-5689, 2005.

[21] M. Tenoutit, N. Maamri, and J. Trigeassou, "An output feedback approach to the design of robust fractional PI and PID controllers," in World Congress IFAC, (Milan, Italy), 2011.

[22] A. Oustaloup, La Commande CRONE. Paris: Hermes, 1991.

[23] C. A. Monje, Y. Q. Chen, B. M. Vinagre, D. Xue, and V. Feliu, Fractional-order systems and controls: Fundamentals and Applications. Berlin: Springer, 2010.

[24] P. Shah and S. Agashe, "Review of fractional PID controller," Mechatronics, vol. 38, pp. 29-41, 2016.

[25] F. Padula and A. Visioli, "Tuning rules for optimal PID and fractionalorder PID controllers," Journal of Process Control, vol. 21, pp. 69-81, 2011.

[26] C. Zhao, D. Xue, and Y. Chen, "A fractional order PID tuning algorithm for a class of fractional order plants," in in Proc. IEEE Int. Mechatronics and Automation Conf., vol. 1, pp. 216-221, 2005.

[27] A. Tepljakov, E. Petlenkov, and J. Belikov, "A flexible MATLAB tool for optimal fractional-order PID controller design subject to specifications," in Proc. European Contr. Conf., (Heifi, China), pp. 4698-4703, 2012.

[28] A. Tepljakpv, E. Petlenkov, J. Belikov, and M. Halas, "Design and implementation of fractional-order PID controllers for a fluid tank system," in Proc. IEEE American Contr. Conf., (Washington, DC, USA), pp. 1777-1782, 2013.

[29] A. Oustaloup, B. Mathieu, and P. Lanusse, "The CRONE control of resonant plants: application to a flexible transmission," European $J$. Contr., vol. 1, pp. 113-121, 1995.

[30] A. Oustaloup, F. Levron, F. Nanot, and B. Mathieu, "Frequency-band complex non-integer differentiator: Characterization and synthesis," IEEE Trans. Circ. Syst. I : Fund. Theory \& Appli., vol. 47, no. 1, pp. $25-40,2000$. 